\title{
A!
}

This is an electronic reprint of the original article.

This reprint may differ from the original in pagination and typographic detail.

Subramaniyam, Nagarajan; Shah, Ali; Dreser, Christoph; Isomäki, Antti; Fleischer, Monika; Sopanen, Markku

Nonlinear plasmonic behavior of nanohole arrays in thin gold films for imaging lipids

Published in:

Applied Physics Letters

DOI:

$10.1063 / 1.5028118$

Published: 04/06/2018

Document Version

Publisher's PDF, also known as Version of record

Please cite the original version:

Subramaniyam, N., Shah, A., Dreser, C., Isomäki, A., Fleischer, M., \& Sopanen, M. (2018). Nonlinear plasmonic behavior of nanohole arrays in thin gold films for imaging lipids. Applied Physics Letters, 112(23), [233109]. https://doi.org/10.1063/1.5028118

This material is protected by copyright and other intellectual property rights, and duplication or sale of all or part of any of the repository collections is not permitted, except that material may be duplicated by you for your research use or educational purposes in electronic or print form. You must obtain permission for any other use. Electronic or print copies may not be offered, whether for sale or otherwise to anyone who is not an authorised user. 


\section{Nonlinear plasmonic behavior of nanohole arrays in thin gold films for imaging lipids}

Nagarajan Subramaniyam, Ali Shah, Christoph Dreser, Antti Isomäki, Monika Fleischer, and Markku Sopanen

Citation: Appl. Phys. Lett. 112, 233109 (2018); doi: 10.1063/1.5028118

View online: https://doi.org/10.1063/1.5028118

View Table of Contents: http://aip.scitation.org/toc/apl/112/23

Published by the American Institute of Physics

\section{Articles you may be interested in}

Molecular beam epitaxy of single-crystalline aluminum film for low threshold ultraviolet plasmonic nanolasers Applied Physics Letters 112, 231904 (2018); 10.1063/1.5033941

Plasmonic nanoparticle lithography: Fast resist-free laser technique for large-scale sub-50 nm hole array fabrication

Applied Physics Letters 112, 223101 (2018); 10.1063/1.5025096

Electromagnetically induced transparency in sinusoidal modulated ring resonator

Applied Physics Letters 112, 234102 (2018); 10.1063/1.5029307

Strong light extraction enhancement using $\mathrm{TiO}_{2}$ nanoparticles-based microcone arrays embossed on III-Nitride light emitting diodes

Applied Physics Letters 112, 231101 (2018); 10.1063/1.5021301

Ultrahigh refractive index sensitivity via lattice-induced meta-dipole modes in flat metallic nanoantenna arrays Applied Physics Letters 112, 223102 (2018); 10.1063/1.5031768

Silicon quantum dots for energetic material applications

Applied Physics Letters 112, 233108 (2018); 10.1063/1.5022587

\section{Conference Proceedings}




\title{
Nonlinear plasmonic behavior of nanohole arrays in thin gold films for imaging lipids
}

\author{
Nagarajan Subramaniyam, ${ }^{1, a)}$ Ali Shah, ${ }^{1}$ Christoph Dreser, ${ }^{2}$ Antti Isomäki, ${ }^{3}$ \\ Monika Fleischer, ${ }^{2}$ and Markku Sopanen ${ }^{1}$ \\ ${ }^{1}$ Department of Electronics and Nanoengineering, Aalto University, P.O. Box 13500, FIN-00076 Aalto, \\ Finland \\ ${ }^{2}$ Institute for Applied Physics and Center LISA ${ }^{+}$, Eberhard Karls University of Tübingen, \\ Auf der Morgenstelle 10 and 15, 72076 Tübingen, Germany \\ ${ }^{3}$ Biomedicum Imaging Unit, Faculty of Medicine, University of Helsinki, P.O. Box 63, FIN-00014 Helsinki, \\ Finland
}

(Received 7 March 2018; accepted 26 May 2018; published online 8 June 2018)

\begin{abstract}
We demonstrate linear and nonlinear plasmonic behaviors of periodic nanohole arrays in thin gold (Au) films with varying periodicities. As expected, the linear optical transmission spectra of the nanohole arrays show a red-shift of the resonance wavelength and Wood's anomaly with increasing hole spacing. The optical transmission and electric near-field intensity distribution of the nanohole arrays are simulated using the finite element method. The nonlinear plasmonic behavior of the nanohole arrays is studied by using picosecond pulsed excitation at near-infrared wavelengths. The characteristic nonlinear signals indicating two-photon excited luminescence (TPEL), sum frequency generation, second harmonic generation, and four-wave mixing (FWM) are observed. A maximum FWM/TPEL signal intensity ratio is achieved for nanohole arrays with a periodicity of $500 \mathrm{~nm}$. Furthermore, the significant FWM signal intensity and contrast compared to the background were harnessed to demonstrate the ability of surface-enhanced coherent anti-Stokes Raman scattering to visualize low concentrations of lipids deposited on the nanohole array with a periodicity of $500 \mathrm{~nm}$. Published by AIP Publishing. https://doi.org/10.1063/1.5028118
\end{abstract}

Nanohole arrays have been closely studied in view of their extraordinary optical transmission (EOT) and high localized electric near-field intensities by several groups. ${ }^{1,2}$ EOT has been demonstrated in the microwave, millimeter wave, and sub-millimeter wave $(\mathrm{THz})$ regions. ${ }^{3,4}$ EOT of nanohole arrays is caused by the interaction of light with surface plasmons (SPs). ${ }^{5}$ SPs are collective oscillations of free electrons at the interface between the metal and the dielectric. The linear optical behavior of SPs and the electric near-field intensities in nanohole arrays have been extensively investigated by varying the hole shape, diameter, and lattice periodicity because of their potential applications in surface enhanced Raman scattering (SERS), photonics, microfluidics, and bio-sensing over the past decade. $^{6-8}$ Recently, the nonlinear plasmonic behavior of nanostructures has shown promising applications, for example, the development of advanced nonlinear nanosources based on nanoemitters, sensors, and storage devices. ${ }^{9}$ Likewise, the second order nonlinear behavior of second harmonic generation $(\mathrm{SHG})\left(\omega_{S H G}=2 \omega_{1}\right)$ and sum frequency generation (SFG) $\left(\omega_{S F G}=\omega_{1}+\omega_{2}\right)$ of metal apertures or nanohole arrays has been studied. ${ }^{10,11}$ While the linear optical properties of nanohole arrays have been investigated in depth over the past decade, the third order nonlinear process of four wave mixing (FWM) in nanohole arrays so far has remained relatively unexplored for potential applications in surface enhanced coherent anti Stokes Raman scattering (SECARS). The degenerate FWM requires two excitation laser beams with frequencies $\omega_{1}$ and $\omega_{2}$ that interact with the sample and generate a signal at a new frequency $\omega_{F W M}=2 \omega_{1}-\omega_{2}$. The same

${ }^{\text {a)} E-m a i l: ~ n a g a r a j a n . s u b r a m a n i y a m @ a a l t o . f i ~}$ principle is used in chemically specific optical imaging in the form of coherent anti-Stokes Raman scattering (CARS) in which two pulsed lasers, referred to as the pump and the Stokes beam, are used to generate the output signal via FWM. By matching the frequency difference $\Delta=\omega_{1}-\omega_{2}$ to a Raman vibrational mode of the target molecule, the CARS signal level can increase by up to five orders of magnitude when compared to the spontaneous Raman signal allowing for high speed microscopic imaging at tolerable excitation laser intensities. ${ }^{12}$ Previously, CARS microscopy has been used to image, e.g., lipid droplets in live biological specimens. ${ }^{13}$ The use of CARS microscopy has focused on carbon-hydrogen $(\mathrm{C}$ $\mathrm{H})$ stretching vibrational modes where lipids exhibit strong abundant $\mathrm{C}-\mathrm{H}$ vibrational modes. ${ }^{14}$ However, due to the nonresonant background, the sensitivity of the CARS method has still been limited in its visualization of nano-sized molecules or cell membranes. ${ }^{15}$ In order to increase the sensitivity, surface enhanced Raman scattering (SERS) has been proposed to improve the detection limit to the single molecular level by exploiting the field enhancement in the presence of metallic nanostructured surfaces. ${ }^{16}$ The CARS signal will be enhanced significantly if the pump or the Stokes excitation wavelengths are in resonance with the localized SPs of the plasmonic nanostructures on which the molecules are positioned. ${ }^{17}$ Recently, CARS (SECARS) has been demonstrated to increase signal sensitivity using a planar $\mathrm{Au}$ surface for lipid imaging. ${ }^{18}$ In this work, we demonstrate the nonlinear plasmonic behavior of nanohole arrays in thin Au films with varying periodicities. Surface plasmon enhanced second and third order responses such as two-photon excited luminescence (TPEL), SFG, SHG, and FWM signals are observed in the nanohole arrays. 
Moreover, we demonstrate that a thin lipid layer deposited on the nanohole array can be visualized by surface plasmon enhanced coherent anti-Stokes Raman scattering.

Focused gallium ion beam (FIB) milling was used to fabricate the nanohole arrays in a $60 \mathrm{~nm}$ thick gold $(\mathrm{Au})$ film on a glass substrate. The periodicity of the arrays was varied from 400 to $500 \mathrm{~nm}$ with a fixed hole diameter of $200 \mathrm{~nm}$. Each sample pattern consists of a $27 \times 27 \mu \mathrm{m}^{2}$ nanohole array. For direct FIB milling of Au films, process parameter selection and optimization were performed by using the guidelines reported in Ref. 19. In order to fabricate the nanohole arrays, the smallest current $(9.7 \mathrm{pA})$ was used at a dwell time of $1000 \mu \mathrm{s}$. The optical transmission properties of the nanohole arrays were characterized in water medium by illuminating with white light through a linear polarizer and a $100 \times$ microscope objective and collecting the transmitted light by an optical spectrometer (QE65000, Ocean optics). The simulations were performed using a commercial finite element method (FEM) software (COMSOL Multiphysics). The model geometry consisted of a $60 \mathrm{~nm}$ thick Au layer with a single hole on top of semi-infinite glass (refractive index $\left.n_{g}=1.5\right)$ immersed in water filling the upper halfspace $\left(n_{w}=1.33\right)$. The edges of the hole were rounded with edge radii of $10 \mathrm{~nm}$ at the top and $5 \mathrm{~nm}$ at the bottom. The values of the dielectric function of gold were taken from Ref. 20. The periodicity was adjusted by the length of this unit cell and by choosing periodic boundary conditions. The experimental setup is replicated by our model. The wave vector is perpendicular to the surface. The excitation power is $1 \mathrm{~W}$ per unit cell, which is far more than in the experiment, but as the linear optical properties are simulated, the enhanced electric field scales linearly with the excitation field. A port that is located three times the period above the structure is used to excite a y-polarized electro-magnetic wave. The transmitted light is collected with a port that is located three times the period below the structure. The area of the ports is the same as the area of the unit cell. The near-field distribution images show the enhancement of the electric field in a plane parallel to the surface at half the height of the Au layer.

Nonlinear optical images and emission spectra were acquired with a multiphoton microscope (Leica TCS SP8 CARS, Leica Microsystems GmbH, Germany). The instrument consists of an inverted microscope equipped with an ultra-short pulsed light source (picoEmerald, APE GmbH, Germany) that produces two synchronous beams at a repetition rate of $80 \mathrm{MHz}$. The Stokes beam at $1064.5 \mathrm{~nm}$ was emitted from a neodymium-vanadate $\left(\mathrm{Nd}: \mathrm{YVO}_{4}\right)$ laser, while the pump/probe beam was generated by an optical parametric oscillator (OPO) tunable in the $780-940 \mathrm{~nm}$ range. The pulse widths were 5-7 ps corresponding to the Raman line width of $2-3 \mathrm{~cm}^{-1}$. The pulses from the two sources were temporally and spatially overlapped in the focal plane of the microscope where the average optical power was $2.5 \mathrm{~mW}$ for the Stokes and $2 \mathrm{~mW}$ for the pump/probe beam. The emission spectra were acquired using an integrated spectrophotometer in the range of $420-700 \mathrm{~nm}$ and with the spectral resolution of $5 \mathrm{~nm}$. Lipid analysis was performed by depositing a drop of cholesterol (Sigma-Aldrich Chemie GmbH, Germany) dissolved in an organic solvent (mixture of chloroform and methanol) on a nanohole array with a periodicity of $500 \mathrm{~nm}$. A thin layer of crystalline cholesterol was formed after evaporation of the solvent. The $\mathrm{C}-\mathrm{H}$ vibrational modes of the lipid were characterized in the range from 2800 to $2960 \mathrm{~cm}^{-1}$ by tuning the pump wavelength between 809 and $820 \mathrm{~nm}$ and keeping the Stokes wavelength fixed at $1064.5 \mathrm{~nm}$. For $\mathrm{CH}_{2}$-sensitive imaging, the excitation beams at $817 \mathrm{~nm}$ and $1064.5 \mathrm{~nm}$ were raster-scanned over the sample area. The emitted radiation was bandpass-filtered using the spectrophotometer at $550-640 \mathrm{~nm}$ for the TPEL and $653-673 \mathrm{~nm}$ for the CARS signal and detected simultaneously in the epi-direction using two hybrid GaAsP detectors (HyD, Leica).

The EOT properties of nanohole arrays with varying diameters, periodicities, and depths have been studied previously by several groups. ${ }^{21,22}$ The transmission maxima of different Bragg resonances satisfy the two-dimensional grating coupling conditions for the SP resonance ${ }^{23}$

$$
\lambda_{S P(i, j)}=\sqrt{\frac{\varepsilon_{d} \varepsilon_{m}}{\varepsilon_{d}+\varepsilon_{m}}} \frac{P}{\sqrt{i^{2}+j^{2}}},
$$

where $P$ is the periodicity of the nanohole array, the integers $(\mathrm{i}, \mathrm{j})$ represent the Bragg resonance orders, and $\varepsilon_{\mathrm{d}}$ and $\varepsilon_{\mathrm{m}}$ are the dielectric functions of the water and metal, respectively. The transmission maxima $(1,1)$ and $(1,0)$ together with the transmission minimum (Wood's anomaly, which occurs when light is diffracted by the grating into an angle of $90^{\circ}$, parallel to the surface) red-shift with increasing periodicity of the nanohole arrays. Figure 1(a) shows a tilted view scanning electron microscopy (SEM) image of a nanohole array in a $60 \mathrm{~nm}$ thick $\mathrm{Au}$ layer. The diameter and periodicity of the nanoholes are $200 \mathrm{~nm}$ and $400 \mathrm{~nm}$, respectively. Figure 1(b) shows the measured (solid lines) optical transmission spectra of the nanohole arrays with the periodicities of 400 , 450 , and $500 \mathrm{~nm}$ and a fixed diameter of $200 \mathrm{~nm}$. The nanohole arrays were immersed in water during the measurement. The simulations (dotted lines) are in good agreement with
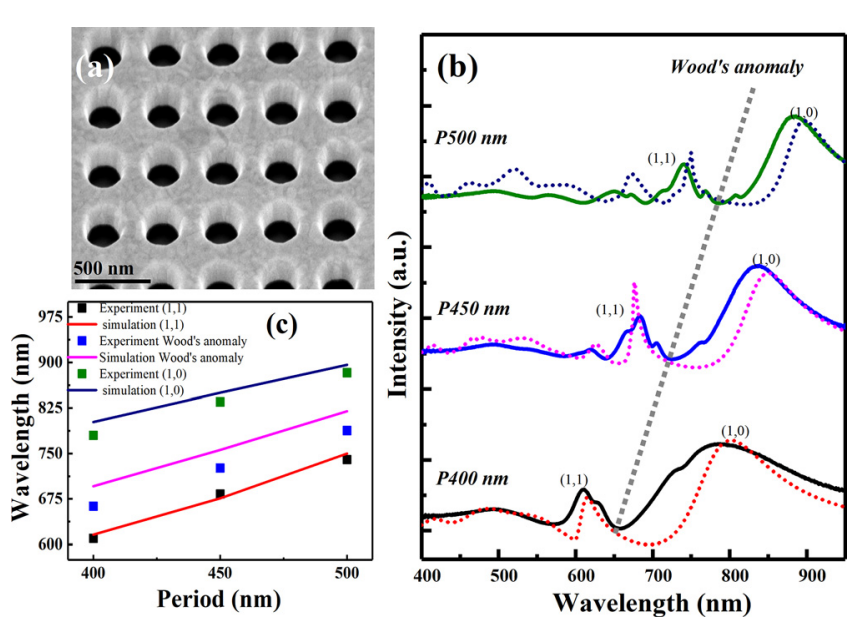

FIG. 1. (a) SEM image of a nanohole array with a periodicity of $400 \mathrm{~nm}$ in the thin Au film. (b) Optical transmission measurements (solid lines) and simulated spectra (dotted lines) of the nanohole arrays with varying periodicities of 400, 450, and $500 \mathrm{~nm}$ in water medium. The spectra are vertically offset for clarity. (c) Optical transmission measurements (solid squares) and simulated data (solid lines) of the resonance peak wavelengths of the $(1,0)$ and $(1,1)$ maxima and Wood's anomaly with respect to the periodicity of the nanohole array. 

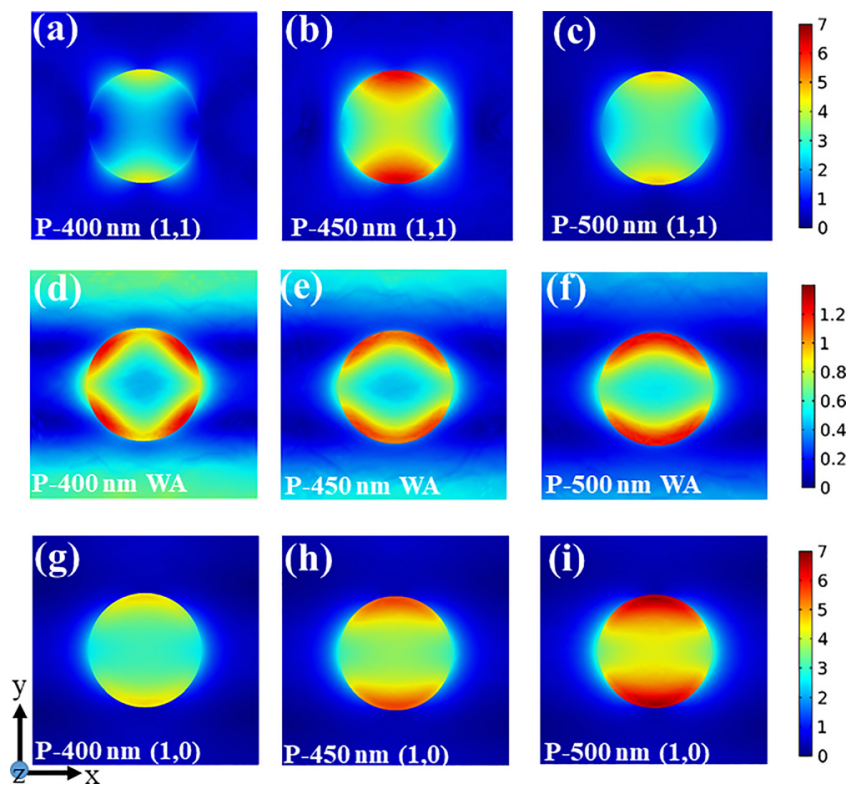

FIG. 2. FEM simulated electric near-field distributions of nanoholes with a diameter of $200 \mathrm{~nm}$ and periodicities of 400, 450, and $500 \mathrm{~nm}$ under excitation with a y-polarized electromagnetic wave at perpendicular incidence. (a)-(c) show the distributions of the electric field enhancement at the wavelengths of the $(1,1)$ resonance peak. (d)-(f) show the distributions at the Wood's anomalies. $(\mathrm{g}),(\mathrm{h})$, and (i) show the distributions at the $(1,0)$ resonance peak.

the measurements. The spectra show two optical transmission maxima corresponding to the surface resonance (SR) peaks $(1,1)$ and $(1,0)$ accompanied by the transmission minimum (Wood's anomaly) for the nanohole arrays. A low peak at $\sim 500 \mathrm{~nm}$ was observed for all nanohole array samples and was attributed to bulk properties of the Au layer. ${ }^{24}$ Figure 1(c) shows the measured (squares) and simulated (solid lines) transmission maxima $(1,1),(1,0)$ and Wood's anomaly wavelengths with respect to the periodicity of the nanohole arrays. The SR wavelengths red-shift linearly with increasing periodicity of the nanohole arrays. To further understand the electric near-field intensity distribution, we performed FEM simulations for the nanohole arrays at the wavelengths of the SP resonance maxima $(1,1)$ and $(1,0)$ and Wood's anomaly. Figure 2 shows the corresponding simulated electric near-field enhancement distributions of the nanohole arrays with varying periodicities. The nanohole arrays were immersed in water medium (refractive index: 1.33). The maximum electric near-field intensity enhancement is observed for the surface resonance $(1,0)$ peaks in the nanohole arrays with $400 \mathrm{~nm}$ and $500 \mathrm{~nm}$ periodicities and for the $(1,1)$ peak in the $450 \mathrm{~nm}$ array.

Panels (a), (b), and (c) of Fig. 3 show the measured TPEL of the nanohole array with a periodicity of $400 \mathrm{~nm}$ with excitation wavelengths of 846,817 , and $780 \mathrm{~nm}$, respectively. The TPEL appears as a broad peak in the range of $550-675 \mathrm{~nm}$ that has a maximum at about $625 \mathrm{~nm}$ for all the excitation wavelengths. The inset shows the relative maximum peak intensities of the TPEL of the nanohole array with respect to the excitation wavelength. The excitation power was kept constant between the measurements. The highest TPEL intensity was observed for an excitation wavelength of $780 \mathrm{~nm}$, and it decreased rapidly as the excitation wavelength was increased to $817 \mathrm{~nm}$. The TPEL properties of Au nanostructures have been studied by several groups. ${ }^{25,26}$ In the TPEL process, electrons from occupied d bands are excited by two-photon absorption to the sp-conduction band and move closer to the Fermi level via an intraband transition within the sp-conduction band. The holes created in the d-band as a result of the two-photon absorption undergo Auger scattering and hole-phonon scattering and subsequently recombine with electrons in the sp-band, emitting the broad luminescence. ${ }^{27}$ Previously, the TPEL properties of Au nanorods, discs, and connected dimers have been studied, showing that the TPEL intensities are dominated by localized surface plasmon resonance (LSPR) wavelengths. ${ }^{28-30}$ The nanohole array with a $400 \mathrm{~nm}$ periodicity shows transmission maxima
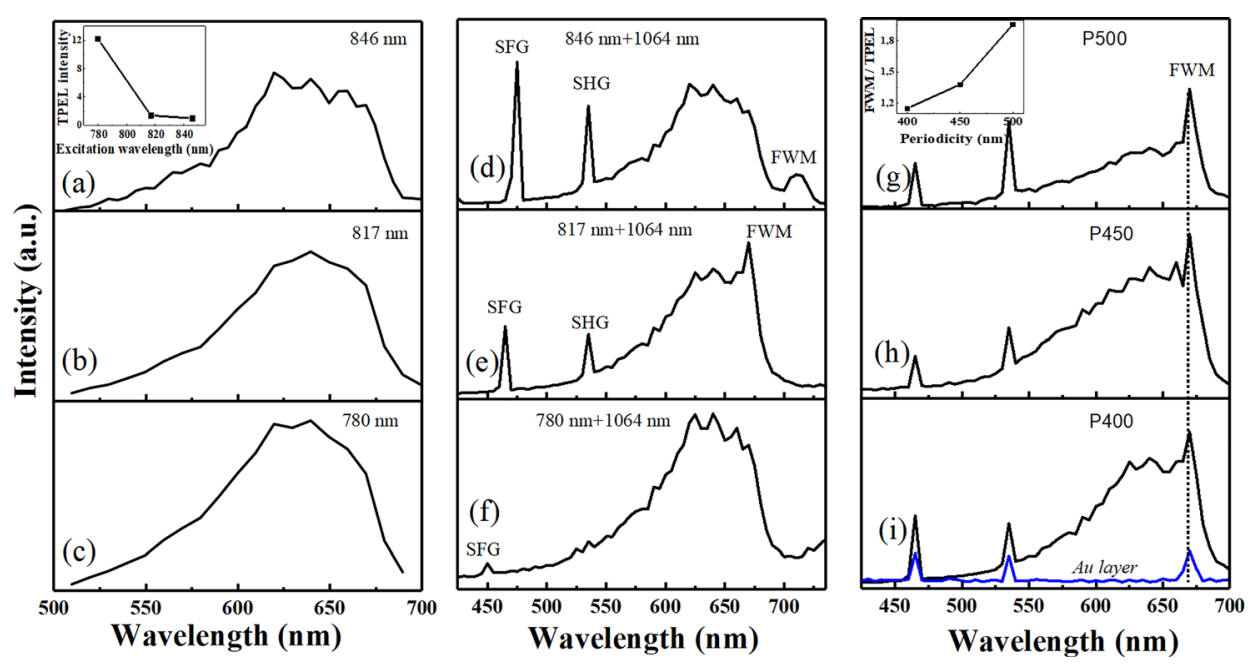

FIG. 3. (a)-(c) Two-photon excited luminescence (TPEL) spectra of the nanohole array with a periodicity of $400 \mathrm{~nm}$ with excitation wavelengths of $846 \mathrm{~nm}$, $817 \mathrm{~nm}$, and $780 \mathrm{~nm}$. The $\mathrm{y}$-axis is scaled between the panels for clarity. The inset shows the TPEL maximum intensity value of the nanohole array with respect to the excitation wavelengths (in relative units). (d)-(f) Nonlinear emission spectra of the 400 nm period nanohole array with a Stokes beam wavelength of $1064.5 \mathrm{~nm}$ in combination with different pump wavelengths of $846 \mathrm{~nm}, 817 \mathrm{~nm}$, and $780 \mathrm{~nm}$. The y-axis is scaled between the panels for clarity. (g)-(i) Nonlinear emission spectra of the nanohole arrays with periodicities of 500, 450, and $400 \mathrm{~nm}$ with a fixed pump wavelength at $817 \mathrm{~nm}$ and a Stokes wavelength of $1064.5 \mathrm{~nm}$. The nonlinear emission spectrum of the unstructured $60 \mathrm{~nm}$ thin Au layer is also shown for comparison (blue line). The inset in (g) shows the ratio between the FWM and TPEL intensities at $665 \mathrm{~nm}$ for the different periodicities, indicating the contrast of the FWM signal over the background. 
of the localized surface plasmon peaks $(1,1)$ and $(1,0)$ at around $610 \mathrm{~nm}$ and $780 \mathrm{~nm}$, respectively [cf. Fig. 1(b)].

Panels (d), (e), and (f) of Fig. 3 show the nonlinear coherent emission spectra of a nanohole array with a periodicity of $400 \mathrm{~nm}$ under excitation with a combination of varying pump laser wavelengths of 846,817 , and $780 \mathrm{~nm}$ and the fixed Stokes laser wavelength of $1064.5 \mathrm{~nm}$. The spectra in (d) and (e) show the SFG, SHG, and FWM signals on top of the broad-band TPEL. On the other hand, the spectrum in (f) shows only a weak SFG peak, and the SHG and the FWM signals are too small to be discerned within the strong TPEL background excited by the pump laser at $780 \mathrm{~nm}$. The SFG and SHG peaks are related to the plasmon-enhanced second order coherent response, and the FWM signal corresponds to the plasmon-enhanced third order coherent response. ${ }^{31}$ The SFG and SHG peak intensities are higher compared to the FWM signal intensity at the longer excitation pump wavelength of $846 \mathrm{~nm}$ related to the increased second order nonlinear response. Previously, the SHG from nanohole arrays in Au layers was studied..$^{32}$ The SHG enhancement from the nanostructure is mainly attributed to the non-centrosymmetric system. The second and third order nonlinear responses are influenced by the geometrical symmetry of the nanohole arrays. The panels (g), (h) and (i) of Fig. 3 show the nonlinear emission spectra of the nanohole arrays with different periodicities upon excitation with the combination of fixed pump $(817 \mathrm{~nm})$ and Stokes $(1064.5 \mathrm{~nm})$ laser lines. The spectra show the SFG peak at $464 \mathrm{~nm}$, the SHG peak at $532 \mathrm{~nm}$, and the FWM peak at $665 \mathrm{~nm}$ together with the broad-band TPEL. In addition, the nonlinear coherent emission spectrum of the unstructured $60 \mathrm{~nm}$ thick Au layer is shown for comparison. The FWM signal and TPEL background signal enhancement were the strongest for the nanohole arrays with the $400 \mathrm{~nm}$ periodicity due to the matching resonance with the Wood's anomaly wavelength at $663 \mathrm{~nm}$. The FWM, SFG, and SHG signal intensities as well as the TPEL background decrease with increasing periodicity of the nanohole arrays. The inset of Fig. 3(g) shows the FWM/TPEL peak maximum intensity ratio with respect to the periodicity. The ratio was determined at $665 \mathrm{~nm}$ by comparing the FWM peak intensity with the TPEL background obtained with $817 \mathrm{~nm}$ excitation alone. The average intensities were measured over the whole array area. The high FWM/TPEL intensity ratio of the nanohole array with the periodicity of $500 \mathrm{~nm}$ is promising for biosensing and lipid imaging applications due to its higher contrast.

To study the role of SECARS of the nanohole arrays for lipid imaging, we deposited a layer of cholesterol on the nanohole array as described in the experimental section. An array with the periodicity of $500 \mathrm{~nm}$ was selected due to the higher FWM/TPEL intensity ratio. Figure 4(a) shows a bright-field reflection image of the cholesterol layers on the nanohole array. Some of the larger crystals with a diameter of $\sim 0.5-1 \mu \mathrm{m}$ appear as dark spots in the image. Figure 4(b) shows an atomic force microscopy (AFM) image of the cholesterol on the nanohole array. The nanoholes are filled by the cholesterol, and the thickness of the cholesterol layer is about $30 \mathrm{~nm}$. Figure 4(c) shows the spontaneous Raman spectrum of cholesterol on the nanohole array measured by a continuous laser excitation at $532 \mathrm{~nm}$. The spectrum shows several vibrational modes, including prominent peaks at

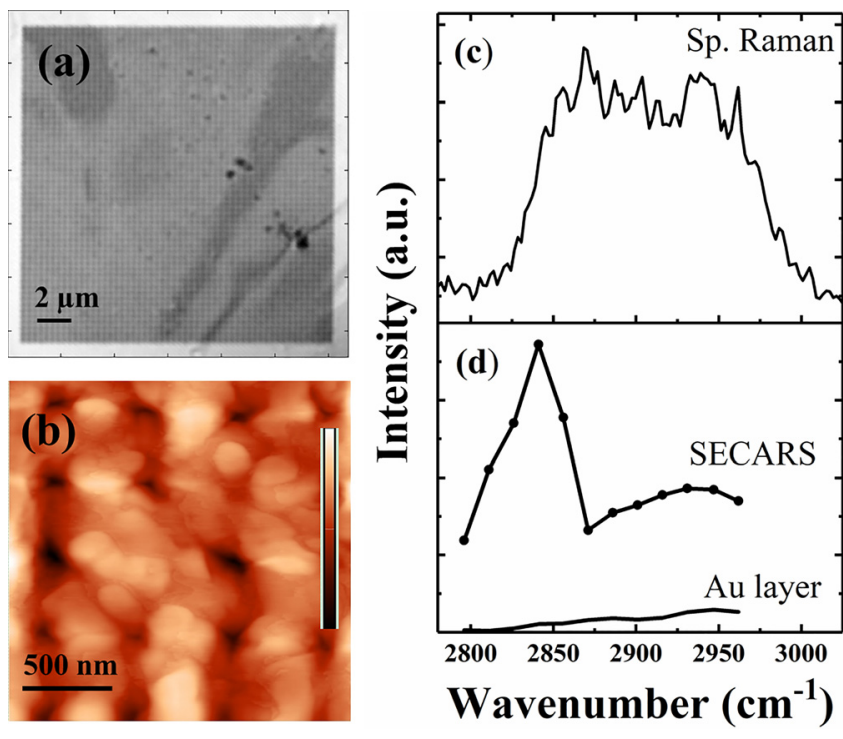

FIG. 4. (a) Bright-field image of the dried cholesterol layer on the nanohole array with a periodicity of $500 \mathrm{~nm}$. (b) Atomic force microscopy (AFM) image of the cholesterol crystals on the nanohole array. The color scale of the image is $30 \mathrm{~nm}$. (c) Spontaneous Raman spectrum of cholesterol on the nanohole array. (d) SECARS spectrum of the cholesterol on the nanohole array. The signal from cholesterol on the Au layer is also shown for comparison.

$2845 \mathrm{~cm}^{-1}, 2855 \mathrm{~cm}^{-1}, 2869 \mathrm{~cm}^{-1}, 2903 \mathrm{~cm}^{-1}, 2943 \mathrm{~cm}^{-1}$, and $2961 \mathrm{~cm}^{-1}$. All of the spectral signatures are C-H stretching vibrational frequency modes of cholesterol in this range. ${ }^{33}$ Figure 4(d) shows the SECARS spectrum of cholesterol on the nanohole array as well as the signal from the cholesterol layer deposited on the plain Au adjacent to the nanohole area. The spectrum measured on the nanoholes shows a distinct peak at $2845 \mathrm{~cm}^{-1}$ corresponding to the $\mathrm{CH}_{2}$ symmetric stretching vibrational frequency mode of cholesterol, thus indicating that the signal is vibrationally sensitive. Outside the nanohole area, only a weak TPEL background with no spectral characteristics was detectable with the same measurement parameters. The significant difference in the CARS signal intensities clearly indicates a strong surface plasmon resonance induced enhancement on the nanohole array with a high FWM/TPEL ratio.

In order to visualize the small cholesterol deposits, we performed SECARS imaging of the $\mathrm{CH}_{2}$ stretching vibrational mode at $2845 \mathrm{~cm}^{-1}$ by using the fixed pump and Stokes wavelengths at 817 and $1064.5 \mathrm{~nm}$, respectively. Figure 5(a) shows the SECARS image of the cholesterol layer on the nanohole array, obtained at the $\mathrm{CH}_{2}$ stretching vibrational mode of $2845 \mathrm{~cm}^{-1}$. The color-coded image shows several bright spots arising from the cholesterol deposits. Figure 5(b) shows a TPEL image of the same area when exciting the sample with the pump beam at $817 \mathrm{~nm}$ alone. The observed TPEL background signal originated from the pump-induced signal of the LSPR-enhanced transitions in the nanohole arrays by electron-hole radiative recombination. Potential TPEL arising from the $1064.5 \mathrm{~nm}$ excitation was below the detection limit. Figure 5(c) shows the SECARS image after TPEL background subtraction. Small deposits of cholesterol in the size range of approximately 300 to $1500 \mathrm{~nm}$ could be visualized close to the nanohole-sample interface with low excitation powers due to the LSPR of the nanoholes. 

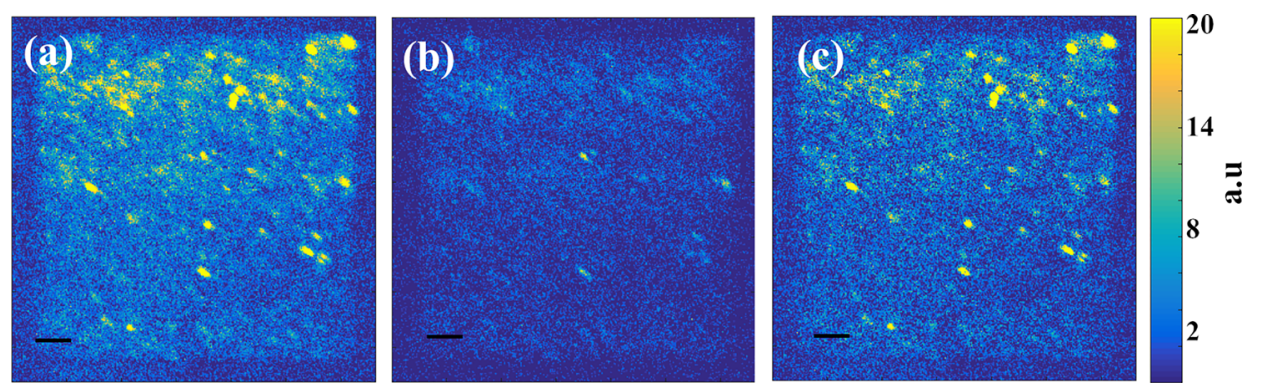

FIG. 5. (a) SECARS image of the cholesterol layer on the nanohole array with a periodicity of $500 \mathrm{~nm}$. (b) TPEL image of the same area. (c) TPEL subtracted SECARS image of the cholesterol layer on the nanohole array. The scale bar is $2 \mu \mathrm{m}$.

We demonstrated linear and nonlinear plasmonic behaviors of periodic nanohole arrays with varying periodicities in thin Au films. As expected from the literature, the EOT of the nanohole arrays showed a red-shift of the surface plasmon resonance wavelength and Wood's anomaly with increasing periodicity. The maximum electric near-field intensity enhancement is observed for the surface plasmon resonance $(1,1)$ peak for the nanohole arrays with $450 \mathrm{~nm}$ periodicity and for the $(1,0)$ peaks for the nanohole arrays with $400 \mathrm{~nm}$ or $500 \mathrm{~nm}$ periodicities, respectively, from simulations using the FEM method. The nonlinear optical signals originating from TPEL, SFG, SHG, and FWM were observed in the nanohole arrays by using a picosecond pulsed excitation laser at near-infrared wavelengths. The highest FWM/TPEL signal intensity ratio was achieved for the nanohole array with a periodicity of $500 \mathrm{~nm}$. Making use of the relatively strong nonlinear signal contrast, SECARS imaging was demonstrated to visualize a low concentration of lipids deposited on the nanohole array with a periodicity of $500 \mathrm{~nm}$.

S.N. acknowledges financial support by the European Cooperation in Science and Technology (COST) Action MP1302 Nanospectroscopy for Short-Term Scientific Mission (STSM) visits to M.F. in Tübingen. C.D. acknowledges financial support through Landesgraduiertenförderung by University of Tübingen and the Ministry of Science, Research and the Arts Baden-Württemberg. The authors thank Professor Elina Ikonen and Simon Pfisterer for providing the cholesterol sample and fruitful discussions. S.N. also thanks N. Chekurov for helping with the FIB process.

${ }^{1}$ T. W. Ebbesen, H. J. Lezec, H. F. Ghaemi, T. Thio, and P. A. Wolff, Nature 391, 667 (1998).

${ }^{2}$ A. G. Brolo, E. Arctander, R. Gordon, B. Leathem, and K. L. Kavanagh, Nano Lett. 4(10), 2015-2018 (2004).

${ }^{3}$ H. Cao and A. Nahata, Opt. Express 12, 1004 (2004).

${ }^{4}$ M. Beruete, M. Sorolla, I. Campillo, J. S. Dolado, L. Martin-Mareno, J. Bravo-Abad, and F. J. Garcia-Vidal, IEEE Trans. Antennas Propag. 53, 1897 (2005).

${ }^{5}$ H. F. Ghaemi, T. Thio, D. E. Grupp, T. W. Ebbesen, and H. J. Lezec, Phys. Rev. B 58, 6779 (1998).

${ }^{6}$ A. E. Certin, D. Etezadi, B. C. Galarreta, M. P. Busson, Y. Eksioglu, and H. Altug, ACS Photonics 2, 1167-1174 (2015).
${ }^{7}$ M. E. Stewart, N. H. Mack, V. Malyarchuk, J. A. N. T. Soares, T. W. Lee, S. K. Gray, R. G. Nuzzo, and J. A. Rogers, Proc. Natl. Acad. Sci. 103, 17143-17148 (2006).

${ }^{8}$ A. Lesuffleur, H. Im, N. C. Lindquist, and S. H. Oh, Appl. Phys. Lett. 90, 243110 (2007).

${ }^{9}$ M. Kauranen and A. V. Zayats, Nat. Photonics 6, 737 (2012).

${ }^{10}$ M. Airola, Y. Liu, and S. Blair, J. Opt. A: Pure Appl. Opt. 7, S118-S123 (2005).

${ }^{11}$ S. Palomba, M. Danckwerts, and L. Novotny, J. Opt. A: Pure Appl. Opt. 11, 114030 (2009).

${ }^{12}$ A. Zumbusch, G. R. Holtom, and X. S. Xie, Phys. Rev. Lett. 82, 4142 (1999).

${ }^{13}$ A. Silve, N. Dorval, T. Schmid, L. M. Mir, and B. A. Attal-Tretout, J. Raman Spectrosc. 43, 644-650 (2012).

${ }^{14}$ T. T. Le, S. Yue, and J. X. Cheng, J. Lipid Res. 51, 3091 (2010).

${ }^{15}$ C. W. Freudiger, W. Min, B. G. Saar, S. Lu, G. R. Holtom, C. He, J. C. Tsai, J. X. Kang, and X. S. Xie, Science 322, 1857 (2008).

${ }^{16}$ K. Kneipp, Y. Wang, H. Kneipp, L. T. Perelman, I. Itzkan, R. Dasari, and M. S. Feld, Phys. Rev. Lett. 78, 1667 (1997).

${ }^{17}$ Y. Zhang, Y. R. Zhen, O. Neumann, J. K. Day, P. Nordlander, and N. J. Halas, Nat. Commun. 5, 4424 (2014).

${ }^{18}$ A. Fast, J. P. Kenison, C. D. Syme, and E. O. Potma, Appl. Opt. 55, 5994 (2016).

${ }^{19}$ N. Chekurov, K. Grigoras, A. Peltonen, S. Franssilla, and I. Tittonen, Nanotechnology 20, 065307 (2009).

${ }^{20}$ P. B. Johnson and R. W. Christy, Phys. Rev. B 6, 4370 (1972).

${ }^{21}$ M. Najiminaini, F. Vasefi, B. Kaminska, and J. J. L. Carson, Appl. Phys. Lett. 100, 043105 (2012).

${ }^{22}$ J. Braun, B. Gompf, T. Weiss, H. Giessen, and M. Dressel, Phys. Rev. B 84, 155419 (2011).

${ }^{23}$ S. H. Chang, S. K. Gray, and G. Schatz, Opt. Express 13, 3150 (2005).

${ }^{24}$ M. Najiminaini, F. Vasefi, B. Kaminska, and J. J. L. Carson, Sci. Rep. 3, 2589 (2013)

${ }^{25}$ P. Melentiev, A. E. Afanasiev, A. A. Kuzin, A. V. Zablotskiy, and V. I. Balykin, Opt. Express 23, 11444 (2015).

${ }^{26}$ P. Reichenbach, A. Horneber, D. A. Gollmer, A. Hille, J. Mihaljevic, C. Schäfer, D. P. Kern, A. J. Meixner, D. Zhang, M. Fleischer, and L. M. Eng, Opt. Express 22, 15484 (2014).

${ }^{27}$ E. Dulkeith, T. Niedereichholz, T. A. Klar, J. Feldmann, G. V. Plessen, D. I. Gittins, K. S. Mayya, and F. Caruso, Phys. Rev. B 70, 205424 (2004).

${ }^{28}$ J. Wang, J. Butet, A.-L. Baudrion, A. Horrer, G. Leveque, O. J. F. Martin, A. J. Meixner, M. Fleischer, P.-M. Adam, A. Horneber, and D. Zhang, J. Phys. Chem. C 120, 17699 (2016).

${ }^{29}$ J. Wang, E. Gurdal, A. Horneber, S. Dickreuter, S. Kostcheev, A. J. Meixner, M. Fleischer, P.-M. Adam, and D. Zhang, Nanoscale 10, 8240 (2018).

${ }^{30}$ A. Bouhelier, R. Bachelot, G. Lerondel, S. Kostcheev, P. Royer, and G. P. Wiederrecht, Phys. Rev. Lett. 95, 267405 (2005).

${ }^{31}$ Y. Wang, C. Y. Lin, A. Nikolaenko, V. Raghunathan, and E. O. Potma, Adv. Opt. Photonics 3, 1 (2011).

${ }^{32}$ B. L. Wang, R. Wang, R. J. Liu, X. H. Lu, J. Zhao, and Z. Y. Li, Sci. Rep. 3, 2358 (2013).

${ }^{33}$ J. Suhalim, C. Y. Chung, M. B. Lilledahl, R. S. Lim, M. Levi, B. J. Tromberg, and E. O. Potma, Biophys. J. 102, 1988 (2012). 